\title{
Dust Mite Allergy and Lifestyle - Indian Perspective
}

\author{
Goutam KS* \\ Department of Zoology, University of Calcutta, India
}

*Corresponding author: Goutam Kumar Saha, Professor of Zoology \& Director, Institute of Agricultural Science, University of Calcutta, 35 Ballygunge Circular Road, Kolkata, India, 700019, Email: gskzoo@gmail.com

\section{Editorial}

Although the prevalence of allergic diseases are more common in westernized and developed countries, the incidence is increasing at a rapid pace in developing countries like India too, becoming doubled, tripled and even quadrupled in the last few decades. However, data in this regard is still fragmentary except few scattered information in Indian context. In a rough estimate, in India, 250 million people are suffering from one or more allergic manifestations. India is the home to around 15-20 million asthmatics and it is increasing day by day. To offer the patients with best possible diagnosis and treatment, the detection of offending allergens are of prime importance. At the same time, early detection of individuals who are genetically at risk of developing allergy to readily available indoor allergens is also an essential element to adopt effective avoidance strategies and to design appropriate therapies. During recent past the most upcoming events related to changes in lifestyle pattern which has come to the surface in an unprecedented manner particularly in developing countries like India are different allergic manifestations due to inhalation of mite laden dust particles. Although house dust has long been known to cause sneezing and wheezing in sensitive subjects, the exact nature of the principal allergen in house dust was unknown for quite a long time. Allergy to house dust mites particularly the genus Dermatophagoides is a common feature in Kolkata metropolitan areas with a steady increase in their incidence in the recent past. A two fold increase in the prevalence of naso-bronchial allergic manifestations has been noted from the urban settings of Kolkata during last few decades. In India, mite associated allergy is a poorly researched area with insufficient quantum of knowledge. It is now well documented that the mites of the genus Dermatophagoides are the most potent allergens in house dust responsible for allergic manifestations. During last more than 30 years, studies on different aspects of house dust mite allergy have been carried out on Kolkata population including entomological, clinical and immunological findings. The study indicates that the house dust sample contains an allergen, secreted and excreted by the house dust mites and inhalation of this allergen along with dust particles initiates allergic manifestations. A total of 53 species belonging to 34 genera, 12 families and 3 orders have been identified so far, 18 species have been recorded for the first time from India and 7 species were identified as new to science. The genus Dermatophagoides alone constituted $60 \%$ of the total acarine fauna, predominated by $D$. pteronyssinus (47\%) followed by D. farina. Both D. pterinyssinus (DP) and D. farina (DF) coexisted in the same habitat and maximum number of mites isolated from an individual dust sample was 13750/gm of dust. Seasonal trend indicates mite count was higher in pre-monsoon and minimum during winter and is abundant in beds than elsewhere in the house and mostly associated with pillows, blankets, mattresses, padded furniture and carpeted floors etc. and preferably feed on human dander. Preliminary screening through Skin Prick Test showed 83\% patients reacted positively towards allergens of mites (either DP or DF), 92\% patients had elevated levels of serum IgE and the mean value higher than control sera $(p<<0.001) .85 \%$ patients of the study group showed allergen specific IgE antibodies against house dust and Dermatophagoides mites. The study indicates that more than $80 \%$ allergic asthma patients residing in Kolkata metropolitan areas are sensitive to dust mites, especially genus Dermatophagoides Spp. as evident from skin prick test and detection of allergen specific IgE antibodies by Immuno Cap system. It is assumed that changes in life style including diet and dietary habits, acquisition of western lifestyle, low standard of indoor environment, increasing air pollution and over all intolerable psychological stress are blamed for such an increased 


\section{International Journal of Zoology and Animal Biology}

occurrence and frequent recurrences of allergic manifestations in Kolkata metropolitan areas. Increase use of padded furniture, sofa sets, heavy curtains, wall to wall carpeting, blankets without any cover instead of traditional quilt, use of foam mattress instead of conventional cotton mattress and soft toys favor the growth and multiplication of house dust mites, which ultimately increases the chances and duration of exposure to those indoor allergens. In more than $70 \%$ cases these may be attributed to the metamorphic change in lifestyle.

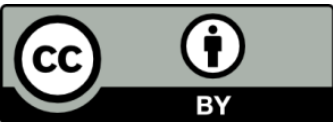

